\title{
Maternal dietary diversity and Nutritional adequacy in relation with anthropometric measurements of newborns at birth: a cohort study in Tehran city
}

Tahereh Karimi

Shahid Beheshti University of Medical Sciences School of Nutrition and Food Technology

Hassan Eini Zinab

Shahid Beheshti University of Medical Sciences School of Nutrition and Food Technology

\section{Zeinab Moslemi}

Shahid Beheshti University of Medical Sciences School of Nutrition and Food Technology

arezoo rezazadeh ( $\square$ arezoo.rezazadeh@gmail.com )

Shahid Beheshti University of Medical Sciences School of Nutrition and Food Technology

\section{Research article}

Keywords: Diet diversity and adequacy, Pregnancy, Neonatal, Anthropometric characteristics, Cohort study

Posted Date: July 17 th, 2020

DOI: https://doi.org/10.21203/rs.3.rs-36658/v1

License: () (i) This work is licensed under a Creative Commons Attribution 4.0 International License. Read Full License

Version of Record: A version of this preprint was published at BMC Pediatrics on March 12th, 2022. See the published version at https://doi.org/10.1186/s12887-021-03102-3. 


\section{Abstract \\ Background}

Maternal dietary intake during pregnancy plays an important role in fetal development and birth outcomes. The aim of the present study was to determine maternal dietary diversity and Nutritional adequacy in relation with anthropometric measurements of newborn at birth as a cohort study in Tehran city.

\section{methods}

This prospective cohort study, was conducted by participation of 585 pregnant women referred to public health centers and hospitals covered by Shahid Beheshti, Tehran and Iran Universities in Tehran City. Using face-to-face interviews, general characteristics were obtained by questionnaire. Pre-pregnancy dietary intake was measured by a 168-item semi-quantitative food frequency questionnaire at the first visit, and dietary intake during pregnancy was measured by 2 non-consecutive 24-hour food recall (one holiday and one regular day) at 31-34 weeks. Maternal height and weight were measured using standard tools and protocol at the first visit, and maternal weight at the end of pregnancy and data related to neonatal anthropometric indices were collected from mothers and neonates health records in the Sib electrical system. By applying SPSS software (version 23) the association was analyzed by linear regression with adjusting for confounding factors. P-value $<0.05$ was considered as significant.

\section{Results}

Mean \pm standard deviation of body mass index (BMI) of pre-pregnancy, pregnancy weight gain, BMI for age z-score (BAZ) at birth of infants were $24.52 \pm 4.12$, $12.16 \pm 6.85 \mathrm{~kg}$ and $-0.61 \pm 1.48$, respectively. Mean \pm SD of the Dietary Diversity Score (DDS) and Mean Adequacy Ratio (MAR) before and during pregnancy were $5.31 \pm 1.11$, vs. $5.23 \pm 1.42$ and $289.85 \pm 113.12$ vs. $371.07 \pm 197.28$, respectively. After adjusting for confounding factors DDS in the third trimester of pregnancy was inversely correlated with WAZ $\left(B=-0.16,95 \% \mathrm{Cl}=-0.23 \_0.30\right)$ and $\mathrm{BAZ}\left(\mathrm{B}=-0.24,95 \% \mathrm{Cl}=-0.06 \_0.42\right)$ at birth, MAR of pre-pregnancy $(\mathrm{B}=$ $\left.-0.001,95 \% \mathrm{Cl}=-0.002 \_0.00\right)$ and in the third trimester of pregnancy $\left(\mathrm{B}=-0.18,95 \% \mathrm{Cl}=-0.35 \_0.004\right)$ were negatively associated with WAZ at birth.

\section{Conclusion}

The findings showed that maternal nutritional status (dietary diversity and nutritional adequacy) before and during pregnancy were correlated with neonatal anthropometric indices at birth.

\section{Background}

Maternal dietary intake during pregnancy plays a vital role on fetus growth and birth outcomes (1-6). Mother's nutritional status is an important marker of infant's survival and health outcomes such as chronic disease risk during their childhood and adult life (7-9). Although animal studies have consistently demonstrated a strong positive relationship between adequate maternal nutrition and birth outcomes, this relationship is much less consistent in humans. This inconsistency maybe due to a confounding effects of number of factors including cultural background, socioeconomic differences(10). Pregnant women are vulnerable groups for malnutrition risk particularly those living developing countries that a significant number of expectant mothers do not receive optimal level of essential nutrients during their pregnancy due to socio-economic constraints, frequent reproductive cycles and poor diet quality $(8,11-14)$.

One of the main manifestation of mother malnutrition is her low weight gain during pregnancy and nonoptimal anthropometric measurements of her newborn. Most of the studies that investigated the association between maternal nutrition with newborn size have approached only weight of the neonatal as a parameter of fetal growth $(15,16)$. It is possible other measurements of fetal size such as length, head circumference, Height-for-Age (HAZ), weight-for-age (WAZ), head circumference for age, body mass index (BMI) may be important in predicting the outcome of fetal growth.

Dietary diversity score (DDS), is a simple, rapid and useful tool used as an indicators for assessing the adequacy of nutrient and energy intake, diet quality and reflects the consumption of various foods between and within each foods group (16-19). Hence, a study was designed to highlight the relationship between maternal dietary diversity and Nutritional adequacy before and during pregnancy and anthropometric measurement of newborn at birth.

\section{Method}

This cohort study was performed on 585 pregnant women referring to public health centers and hospitals under-coverage of Shahid Beheshti, Tehran and Iran universities between Decembers 2015_ may 2018. Pregnant women in their first trimester of pregnancy were recruited from public health facilities for first interview. Criteria for entering the study included being at $<22$ weeks of gestation, tending to cooperate with the project and for newborns including live and healthy cases born from studied pregnant mothers. Exclusion criteria included the compliance of a pregnant mother with a particular diet, unwillingness to continue the collaboration with the project, abortion and miscarriage, over and under reporting of energy intake outside the range of mean \pm 3 standard deviation and birth of infants with severe conditions such as physical or mental retardation.

The data collected were pregnancy history, dietary intake (i.e. Food Frequency Questionnaire (FFQ)), socioeconomic status, and other mother-related factors related to birth weight. The second interview included two phone calls to 24-hour dietary recall (24-h DR) information from mother at third trimester. The third stage of data collection included gathering mother and infant information from health facilities routine registration system at the end of third trimester of mother and new born birth. 
Sample size was calculated by use of the Open Epi Kelsey statistical software OpenEpi (20) according to the study of Melby et al (21) and assumptions: a $95 \%$ significance level.

\section{Dietary assessment}

Dietary intake data were collected by trained nutritionist. Information about first stage nutritional intake was assessed using a validated semi-quantitative FFQ with 168 food items (with standard serving sizes) through face to face interviews. The FFQ was designed according to Willet's method (22). The reliability and validity of the questionnaire was evaluated earlier using a sample of adult women in Tehran(23). For each food item, participants reported the consumption frequency according to suggested portion size during the past year. Dietary intake of mothers at third trimester of pregnancy measured using two nonconsecutive 24_h recall including food consumption for a typical week day and one weekend day collected at 31-34 ${ }^{\text {th }}$ weeks of their gestation. Afterward, reported dietary intake by both dietary assessment methods were converted to daily consumption in grams by using household measures(24). Mean intake of two 24-h DR was considered for second stage analysis. Total daily energy intake was calculated using the information from USDA food composition table (USDA, Release 11, 1994) adapted for Iranian food products(25).

\section{DDS and nutritional adequacy assessment}

Kant et al have developed a method for scaling dietary diversity using a variety of food groups(26). In this method, the categorization of food groups according to the definition of the food pyramid, were used 5 groups of bread and cereals, vegetables, fruits and dairy products meat and its alternatives. The main groups were divided into 23 subgroups ( 7 for bread and cereals, 7 for vegetables, 2 for fruits, 3 for dairy products and 4 for meat and alternatives). These subgroups were defined to include diversity of food items in each food group of the pyramid (27). The division of bread and cereals into seven subgroups shows the importance of diversity in the consumption of cereal-based foods. In order to be considered as a consumer of a food subgroup, at least half of the defined portion of that food must be consumed in accordance with the definitions of the quantitative indicators of the food pyramid in the 2 days of recall. Certainly, a higher score and close to 10 indicates a better compliance with the diversity principle based on the recommendations of the food pyramid. Of course, the probability that a person will use all 23 subgroups of the pyramid within 2 days is very low and studies have shown that perhaps only $10 \%$ of the population can use all 23 subgroups within two days(28).

Nutrient adequacy ratios (NARs) were calculated for the intake of energy and the 11 nutrients (vitamin A, riboflavin, thiamine, vitamin C, calcium, iron, zinc, Phosphorus, magnesium, protein and potassium) based on the recommended daily allowance (RDA) for that nutrient. In the present study, it was used the medical institution's RDA for the different age groups of pregnant women(29). The average of the food adequacy ratio was based on the formula in the KrebsSmith study and the indicator was calculated by dividing the amount of nutrients and energy consumed by the amount of their recommended amount(30). MAR was also calculated as the sum of NARs for all assessed nutrients divided by the number of assessed nutrients. To assess an overall estimate of the nutritional adequacy a mean adequacy ratio (MAR) was calculated from the 11 NARs according to the following formula:

$$
M A R=\frac{\sum N A R}{\text { Number of nutrients }}
$$

\section{Assessment of other variables}

Data on maternal demographic (age, marital status, pre-marital familial relationship with spouse, number of family members, religion, ethnicity, and nationality), socioeconomic status (education, employment status, household income) were collected by a valid questionnaire (31). Other variables included physical activity assessed by International Physical Activity Questionnaire validated for Iranians (IPAQ) (32), general health status assessed by validated GHQ28 questionnaire(33). Pregnancy complications, past medical history and abortion, number of prenatal care, pregnancy number, and gestational age were also collected by question and referring to the electronic health records (Samaneh Sib).

\section{Maternal and Neonatal Anthropometric Measures}

Pre-pregnancy weight was obtained from the health registry system. Mother's weight and height were measured at first interview, as well. Weight was measured to the nearest $100 \mathrm{~g}$ without shoes and with minimal clothes. Height was measured to the nearest 1 mm without shoes and standing straight with normal position of shoulders. Mother's Body Mass Index was calculated as weight (in kilograms) divided by height (in meters) squared. Mother's weight at third trimester obtained from health records after making adjustments for differences in measurement tools. The health facility scales were calibrated using research team scales. The final Information on infant anthropometric measurements and gestational age were obtained from Samaneh Sib. Infant WAZ, HAZ and BAZ were calculated using gender-specific reference database form the World Health Organization (WHO) AnthroPlus for personal computers software, version 1.0.4)(34).

\section{Statistical analysis}

Data was analyzed using SPSS software (version 21). Both univariate and multivariate analysis were conducted. For univariate analysis, frequency and percentage or mean and standard deviation was used depending on the measurement type. The relationships between maternal dietary diversity and nutritional adequacy before and during pregnancy with anthropometric measurements of newborn at birth were analyzed at both pre-pregnancy and third trimester. Unadjusted and adjusted linear regression models were used to assess the relationships. Dependent variable for both unadjusted and adjusted linear models was birth weight.

From the total 691 women recruited at first stage interview, 585 women completed all questionnaires of the first stage (figure 1) of whom, 345 were available/willing to participate at second interview and completed the 24-h recall. At the third stage, information of 337 infants anthropometric 
measurements (considering all participating mothers at the first stage who delivered alive and healthy baby) at birth were obtained from electronic health records.

\section{Results}

Socioeconomic and demographic characteristics of the samples are presented in Table 1. Most of mothers were in younger age, housewives with low or medium educational level, living in household with size 4 or less, and about one-third of them had no prior familial relationship with their husband and more than two thirds of them lived in households with lower than the mean monthly income.

Maternal and neonatal anthropometric indices, energy intake, diversity score, and nutritional adequacy of the women studied are shown in Table 2. The results show that the average energy intake of mothers at the third trimester of pregnancy was lower than pre-pregnancy. The mean score of mother's dietary diversity was also reduced during pregnancy, but the mothers' average nutritional adequacy was increased in the third trimester.

Mean scores of food groups according to the total DDS tertiles of pre-pregnancy and third trimester of gestation of studied mothers are presented at table 3 . Findings showed that there was a significant difference between pre-and post-pregnancy dietary diversity for bread and cereals, meat, dairy and vegetables. But for the fruit group, women in the first tertile had a significant difference with women in the second and third tertiles, but no significant difference was found between the second and third tertiles.

Association of before and during pregnancy DDS with infants' anthropometric indices is shown in Table 4. The results showed that there was no significant relationship between pre-pregnancy maternal DDS and neonatal anthropometric indices with moderating effect of confounding variables. But there was a significant inverse relationship between maternal DDS during pregnancy and weight, WAZ and BAZ of neonate.

According to the study findings, the mean carbohydrate, PUFA, sodium, vitamins B6, B12, and vitamin C intake of pre-pregnancy were significantly higher than the third trimester. While, the mean intake of protein, cholesterol, MUFA, iron, calcium, zinc and magnesium was increased significantly during pregnancy (Table 5).

The association between maternal pre-pregnancy and pregnancy nutritional adequacy and infants' anthropometric indices is shown in Table 5. The results showed that there was a significant inverse relationship between maternal nutritional adequacy (both pre-pregnancy and pregnancy status) and weight and WAZ at birth. Head Circumference was inversely associated with pre-pregnancy DDS only in crude model.

\section{Discussion}

To our knowledge, this is the first prospective cohort study to examine the relationship of maternal dietary diversity and nutritional adequacy in both prepregnancy and pregnancy condition with anthropometric measurements of infants at birth. In our study, there was no significant association between prepregnancy maternal DDS and infant anthropometric indices at birth, but after adjusting for maternal and the neonatal confounders, maternal DDS (in gestational stage) and MAR (in both stages of mother`s intake assessment) were negatively associated with Weight and BAZ.

Total DDS of mothers in pre-pregnancy and pregnancy states were 5.31 and 5.23, respectively. The pre-pregnancy score of the studied women was lower than the amount reported in a cross-sectional study in Tehranian women aged 18-80 years that the mean DDS was 6 (35). In two study in Bangladesh (36, 37)the mean women diet diversity score (WDDS) of participants who were in the reproductive age was 4.3 and 3.8 , respectively that was lower than present study.

Dietary intake analysis of studied mothers in both state (pre-pregnancy and third trimester of pregnancy) showed that those with higher score for DDS had higher score for intake of all main component of DDS (bread and cereals, meats, dairy, vegetables and fruit groups). In one study that examined WDDS during pregnancy, women with highest total score obtained higher dairy, animal-source foods, and vitamin A rich fruits \&vegetables scores than those with less total $\operatorname{WDDS}(16)$.

In this prospective cohort study, we observed that maternal DDS during pregnancy was negatively associated with weight, WAZ and BAZ in birth after adjusting for confounding factors. Consistent with this study, the results of a cohort study showed that adequate dietary diversity during pregnancy and greater consumption of dairy, fruits, vegetables, animal foods such as meat and eggs were associated with lower risk of low birth weight (LBW)(16). Another study showed that Individual Dietary Diversity Score (IDDS) had a significant inverse relationship with LBW incidence $(O R=0.43, P=0.014)(10)$. Nonetheless, a randomized controlled trial in India showed that taking of more dairy products, fruits and vegetables before and during pregnancy through a specially formulated snack doesn't affect birth weight(38). In another study WDDS of $\geq 4$ food groups during pregnancy was associated with lower risk of maternal anemia, low birth weight (LBW), preterm birth (PTB)(16) .

In current study, beside maternal and neonatal confounders, adjusting socioeconomic status had an important role in significance of findings. A previous study confirmed that socioeconomic factors, especially household income and education, play a crucial role in women's dietary diversity and wealthy families and literate women had significantly higher dietary diversity scores(36).

The findings of the present study show that, despite high number of mothers were at the lowest tertile of pre-pregnancy DDS, their frequency at the third tertile was increased in pregnancy stage. Perhaps, one of the possible reasons for increasing the DDS of pregnant mothers was due to receive nutritional counseling from primary health centers/hospitals and increasing their sensitivity to follow the nutritional recommendations during this susceptible period. On the other hand, high percentage of newborns had birth weight in the normal range of 2500 to $4000 \mathrm{~g}$ that may be related to increasing mothers' dietary diversity in the third trimester of pregnancy and improving their weight gain resulted to a normal birth weight range of newborns. However, few studies have examined maternal dietary diversity during pregnancy and its association with neonatal anthropometric indices, and this needs further investigation in future studies

Page $4 / 12$ 
Generally dietary diversity improves the intake of micronutrients and leads to improved pregnancy outcomes. Particularly in the second trimester of pregnancy, oxidative stress is reached to the highest level and may lead to inflammation and effect on pregnancy outcomes. Obtaining higher dietary diversity score means that variety of antioxidant rich food groups are eaten that may protect against oxidative stress (16).

In the present study, there was no significant relationship between maternal nutritional adequacy before and during pregnancy with neonatal anthropometric measuresat birth. After adjusting for maternal and neonatal confounders, a reverse significant relationship was observed between maternal's nutritional adequacy whit birth weight and WAZ at birth. In a longitudinal cohort study conducted in Australia, women in the highest quartile of MAR had lower fat and saturated fatty acids and higher protein, carbohydrate, and fiber intake compared with women in the lowest quartile. However, there was not any information of their pre-pregnancy intake (39). In another study examining maternal nutritional adequacy during pregnancy based on RDA, some micronutrients such as vitamin C, vitamin A, potassium, iron and selenium levels were lower than recommended (40).

The present study showed that the average intake of iron, iodine, vitamin B6, vitamin E and vitamin D was lower than recommended dietary allowance (RDA) in pregnant women but the mean calcium intake was 1.4 times higher than RDA. In one study examining the nutrient intake of mothers during pregnancy, the findings showed that mothers had lower vitamin E, folate, magnesium, and iron intake than recommended DRI values(41). In the Australian study, high percentage of women receiving inadequate calcium, folate, magnesium, and potassium and vitamin E indicating lower nutrient intake than pre-pregnancy RDI. However, these studies did not examine the relationship between maternal nutritional adequacy and neonatal anthropometric indices. In addition, other large studies focusing on maternal nutritional adequacy during pregnancy have also examined only one single nutrient $(42,43)$. In a study aimed investigating the effect of pre-pregnancy BMI, energy and nutritional supplements on neonatal body composition, the portion of mother`s carbohydrate from total energy intake was positively related with infant adipose tissue(44).

It seems that high intake of calcium in present study may possibly be associated with reduced risk of overweight and obesity. Apparently, the mechanism of this effect is related to the reduction of PTH and 1, 25-Dihydroxyvitamin D which leads to inhibition of lipogenesis and increase lipolysis and ultimately increase in fecal fat excretion due to the formation of soaps(45). Perhaps this is a reason for the negative relationship seen in this study. However, this reverse association may be attributed to the fact that mothers with higher adequacy of the diet are at the normal range of weight that affects to gain weight at the normal range during gestation and to deliver a baby with normal birth weight.

The main strength of this study is the information was gathered in a prospective cohort and mothers were followed up during pregnancy until delivery. Also, in order to assess the nutritional status of the mothers, two dietary intake methods were used. 24-hour recalls as a gold standard method reflected actual intake of pregnancy stage and FFQ assessed the usual dietary intake of women before deciding to pregnancy. Dietary intake assessments have some limitations such as measurement error, misreporting of dietary intake specially under reporting for unhealthy items by obese mothers $(46,47)$.

\section{Conclusion}

Overall, the findings of the study indicate that there is a relationship between maternal dietary diversity during pregnancy and the maternal nutritional adequacy before and during pregnancy with neonatal anthropometric indices at birth. In general, a mother's nutritional status may have important implications for infant health.

\section{Abbreviations}

FFQ: Food Frequency Questionnaire; BMI:Body Mass Index; WAZ:Weight for Age Z-score; HAZ:Height for Age Z-score; BAZ:BMI for Age Z-score; HCZ:Head Circumference for age Z-score; LBW:Low Birth Weight; MAR:Mean Adequacy Ratio; NAR:Nutrient Adequacy Ratio; DDS:Dietary Diversity Scores; RDA:Recommended Dietary Allowances

\section{Declarations}

\section{Ethics approval and consent to participate}

The study was approved by the Research Ethics Committee of Shahid Beheshti Medical

University of Iran, under protocol number IR.SBMU.RETECH.REC.1398.008 written informed consent was obtained from the studied mothers for participation.

\section{Consent for publication}

Written informed consent was obtained from the studied mothers to publish the findings

\section{Availability of data and materials:}

The data used for this study could not be shared publicly. However all data and outputs are privately available upon request of the BMC pediatrics journal.

\section{Competing interests}

The authors declare that they have no conflict of interest.

\section{Funding:}

There is no funding source to declare. 


\section{Acknowledgments}

The authors wish to thank all who participated in or collaborated with current study.

\section{Authors' contributions}

AR and H.E.Z designed and supervised the whole procedures of the study. T.K and Z.M collected and analyzed data. A.R and T.K prepared the manuscript. H.E.Z reviewed and edited the final

\section{References}

1. Hjertholm KG, Iversen PO, Holmboe-Ottesen G, Mdala I, Munthali A, Maleta K, et al. Maternal dietary intake during pregnancy and its association to birth size in rural Malawi: A cross-sectional study. Matern Child Nutr. 2018;14(1):e12433.

2. Horan MK, McGowan CA, Gibney ER, Donnelly JM, McAuliffe FM. Maternal low glycaemic index diet, fat intake and postprandial glucose influences neonatal adiposity-secondary analysis from the ROLO study. Nutrition journal. 2014;13(1):78.

3. Kizirian N, Markovic T, Muirhead R, Brodie S, Garnett S, Louie J, et al. Macronutrient balance and dietary glycemic index in pregnancy predict neonatal body composition. Nutrients. 2016;8(5):270.

4. Sengpiel V, Elind E, Bacelis J, Nilsson S, Grove J, Myhre R, et al. Maternal caffeine intake during pregnancy is associated with birth weight but not with gestational length: results from a large prospective observational cohort study. BMC Med. 2013;11(1):42.

5. Timmermans S, Steegers-Theunissen RP, Vujkovic M, den Breeijen H, Russcher H, Lindemans J, et al. The Mediterranean diet and fetal size parameters: the Generation R Study. Br J Nutr. 2012;108(8):1399-409.

6. Chen L-W, Aris IM, Bernard JY, Tint M-T, Colega M, Gluckman PD, et al. Associations of maternal macronutrient intake during pregnancy with infant BMI peak characteristics and childhood BMI. Am J Clin Nutr. 2017;105(3):705-13.

7. Brantsæter AL, Birgisdottir BE, Meltzer HM, Kvalem HE, Alexander J, Magnus P, et al. Maternal seafood consumption and infant birth weight, length and head circumference in the Norwegian Mother and Child Cohort Study. Br J Nutr. 2012;107(3):436-44.

8. Shaikh F, Zeeshan F, Hakeem R, Basit A, Fawwad A, Hussain A. Maternal dietary intake and anthropometric measurements of newborn at birth. The Open Diabetes Journal. 2014;7(1):14-9.

9. Walsh JM, McGowan CA, Mahony R, Foley ME, McAuliffe FM. Low glycaemic index diet in pregnancy to prevent macrosomia (ROLO study): randomised control trial. Bmj. 2012;345:e5605

10. Saaka M. Maternal dietary diversity and infant outcome of pregnant women in Northern Ghana. International Journal of Child Health Nutrition. 2013;1(2):148-56.

11. Lee SE, Talegawkar SA, Merialdi M, Caulfield LE. Dietary intakes of women during pregnancy in low-and middle-income countries. Public Health Nutr. 2013;16(8):1340-53.

12. Wrottesley S, Lamper C, Pisa P. Review of the importance of nutrition during the first 1000 days: maternal nutritional status and its associations with fetal growth and birth, neonatal and infant outcomes among African women. Journal of developmental origins of health disease. 2016;7(2):144-62.

13. Worku BN, Abessa TG, Wondafrash M, Vanvuchelen M, Bruckers L, Kolsteren P, et al. The relationship of undernutrition/psychosocial factors and developmental outcomes of children in extreme poverty in Ethiopia. BMC Pediatr. 2018;18(1):45.

14. Kassa GM, Muche AA, Berhe AK, Fekadu GA. Prevalence and determinants of anemia among pregnant women in Ethiopia; a systematic review and metaanalysis. BMC hematology. 2017;17(1):17.

15. Diemert A, Lezius S, Pagenkemper M, Hansen G, Drozdowska A, Hecher K, et al. Maternal nutrition, inadequate gestational weight gain and birth weight: results from a prospective birth cohort. BMC Pregnancy Childbirth. 2016;16(1):224.

16. Zerfu TA, Umeta M, Baye K. Dietary diversity during pregnancy is associated with reduced risk of maternal anemia, preterm delivery, and low birth weight in a prospective cohort study in rural Ethiopia. Am J Clin Nutr. 2016;103(6):1482-8.

17. Melaku YA, Gill TK, Taylor AW, Adams R, Shi Z, Worku A. Associations of childhood, maternal and household dietary patterns with childhood stunting in Ethiopia: proposing an alternative and plausible dietary analysis method to dietary diversity scores. Nutrition journal. 2018;17(1):14.

18. Jiang W, Mo M, Li M, Wang S, Muyiduli X, Shao B, et al. The relationship of dietary diversity score with depression and anxiety among prenatal and postpartum women. Journal of Obstetrics Gynaecology Research. 2018;44(10):1929-36.

19. Gicevic S, Gaskins AJ, Fung TT, Rosner B, Tobias DK, Isanaka S, et al. Evaluating pre-pregnancy dietary diversity vs. dietary quality scores as predictors of gestational diabetes and hypertensive disorders of pregnancy. PloS one. 2018;13(4):e0195103.

20. https:/.

21. Melby MK, Yamada G, Surkan PJ. Inadequate gestational weight gain increases risk of small-for-gestational-age term birth in girls in Japan: A populationbased cohort study. American Journal of Human Biology. 2016;28(5):714-20.

22. Willett W. Nutritional epidemiology: Oxford University Press; 2012.

23. Asghari G, Rezazadeh A, Hosseini-Esfahani F, Mehrabi Y, Mirmiran P, Azizi F. Reliability, comparative validity and stability of dietary patterns derived from an FFQ in the Tehran Lipid and Glucose Study. British journal of nutrition. 2012;108(6):1109-17.

24. Ghaffarpour M, Houshiar-Rad A, Kianfar H. The manual for household measures, cooking yields factors and edible portion of foods. Tehran: Nashre Olume Keshavarzy. 1999;7:213. 
25. Azar M, Sarkisian E. Food Composition Table of Iran. Tehran: National Nutrition and Food Research Institute; 1980.

26. Kant AK, Schatzkin A, Ziegler RG. Dietary diversity and subsequent cause-specific mortality in the NHANES I epidemiologic follow-up study. J Am Coll Nutr. 1995;14(3):233-8.

27. USDA`S Food Guide Pyramid Booklet editor. Washington, DC: US Department of Agriculture. 1-1. 1996.

28. Haines PS, Siega-Riz AM, Popkin BM. The Diet Quality Index revised: a measurement instrument for populations. J Am Diet Assoc. 1999;99(6):697-704.

29. Food NB, Academies loM,N, Food, Nutrition Board loM, National Academies. Dietary Reference Intakes (DRIs): estimated average requirements. National Academies. 2011;1997.

30. Krebs-Smith SM, Smiciklas-Wright H, Guthrie HA, Krebs-Smith J. The effects of variety in food choices on dietary quality. J Am Diet Assoc. 1987;87(7):897-903.

31. Rezazadeh A, Omidvar N, Eini-Zinab H, Ghazi-Tabatabaie M, Majdzadeh R, Ghavamzadeh S, et al. Major dietary patterns in relation to demographic and socio-economic status and food insecurity in two Iranian ethnic groups living in Urmia. Iran Public health nutrition. 2016;19(18):3337-48.

32. Vasheghani-Farahani A, Tahmasbi M, Asheri H, Ashraf H, Nedjat S, Kordi R. The Persian, last 7-day, long form of the International Physical Activity Questionnaire: translation and validation study. Asian journal of sports medicine. 2011;2(2):106.

33. SERUM MAGNESIUM LEVELS AND THE INCIDENCE OF LOW-BIRTH-WEIGHT INFANTS A CASE-CONTROL STUDY SALEHINIYA H, MOHAMADKHANI SL, SABET BS. THE RELATIONSHIP OF MATERNAL. SERUM MAGNESIUM LEVELS AND THE INCIDENCE OF LOWBIRTH-WEIGHT INFANTS: A CASE-CONTROL STUDY. 2014.

34. Organization WH. WHO AnthroPlus for personal computers manual: software for assessing growth of the world's children and adolescents. Geneva: WHO; 2009. Google Scholar. 2016.

35. Mirmiran P, Azadbakht L, Azizi F. Dietary diversity within food groups: an indicator of specific nutrient adequacy in Tehranian women. Journal of the American college of nutrition. 2006;25(4):354-61.

36. Harris-Fry H, Azad K, Kuddus A, Shaha S, Nahar B, Hossen M, et al. Socio-economic determinants of household food security and women's dietary diversity in rural Bangladesh: a cross-sectional study. Journal of Health Population Nutrition. 2015;33(1):2.

37. Arsenault JE, Yakes EA, Islam MM, Hossain MB, Ahmed T, Hotz C, et al. Very low adequacy of micronutrient intakes by young children and women in rural Bangladesh is primarily explained by low food intake and limited diversity. J Nutr. 2012;143(2):197-203.

38. Potdar RD, Sahariah SA, Gandhi M, Kehoe SH, Brown N, Sane H, et al. Improving women's diet quality preconceptionally and during gestation: effects on birth weight and prevalence of low birth weight-a randomized controlled efficacy trial in India (Mumbai Maternal Nutrition Project). Am J Clin Nutr. 2014;100(5):1257-68.

39. Looman M, Schoenaker DA, Soedamah-Muthu SS, Mishra GD, Geelen A, Feskens EJ. Pre-pregnancy dietary micronutrient adequacy is associated with lower risk of developing gestational diabetes in Australian women. Nutr Res. 2019;62:32-40.

40. Horan MK, McGowan CA, Gibney ER, Donnelly JM, McAuliffe FM. The association between maternal dietary micronutrient intake and neonatal anthropometry-secondary analysis from the ROLO study. Nutrition journal. 2015;14(1):105.

41. Pinto E, Barros H, dos Santos Silva I. Dietary intake and nutritional adequacy prior to conception and during pregnancy: a follow-up study in the north of Portugal. Public Health Nutr. 2009;12(7):922-31.

42. van Eijsden M, Hornstra G, van der Wal MF, Vrijkotte TG, Bonsel GJ. Maternal $n-3, n-6$, and trans fatty acid profile early in pregnancy and term birth weight: a prospective cohort study. Am J Clin Nutr. 2008;87(4):887-95.

43. Alwan NA, Cade JE, Greenwood DC, Deanfield J, Lawlor DA. Associations of maternal iron intake and hemoglobin in pregnancy with offspring vascular phenotypes and adiposity at age 10: findings from the Avon Longitudinal Study of Parents and Children. PloS one. 2014;9(1):e84684.

44. Pereira-da-Silva L, Cabo C, Moreira AC, Virella D, Guerra T, Camoes T, et al. The adjusted effect of maternal body mass index, energy and macronutrient intakes during pregnancy, and gestational weight gain on body composition of full-term neonates. Am J Perinatol. 2014;31(10):875-82.

45. Alomaim H, Griffin P, Swist E, Plouffe LJ, Vandeloo M, Demonty I, et al. Dietary calcium affects body composition and lipid metabolism in rats. PloS one. 2019;14(1):e0210760.

46. Moran L, McNaughton S, Sui Z, Cramp C, Deussen A, Grivell R, et al. The characterisation of overweight and obese women who are under reporting energy intake during pregnancy. BMC Pregnancy Childbirth. 2018;18(1):204.

47. Poslusna K, Ruprich J, de Vries JH, Jakubikova M, van't Veer P. Misreporting of energy and micronutrient intake estimated by food records and 24 hour recalls, control and adjustment methods in practice. Br J Nutr. 2009;101(S2):73-85.

\section{Tables}

Table 1: Socioeconomic and demographic characteristics of the sample at first phase of the study 


\begin{tabular}{|c|c|c|}
\hline Characteristics & & Frequency (Percent) \\
\hline \multirow[t]{3}{*}{ Mother's Age (years) } & $<18$ & $17(2.9 \%)$ \\
\hline & $18-35$ & $464(79.3 \%)$ \\
\hline & $>35$ & $104(17.8 \%)$ \\
\hline \multirow[t]{3}{*}{ Mother Education } & Low & $176(30.1 \%)$ \\
\hline & Medium & $249(42.5 \%)$ \\
\hline & University degree & $160(27.4 \%)$ \\
\hline \multirow[t]{2}{*}{ Employment Status of the Mother } & housewife & $532(90.9 \%)$ \\
\hline & Employed & $53(9.1 \%)$ \\
\hline \multirow[t]{2}{*}{ Prior Familial Relationship } & Relative & $204(34.9 \%)$ \\
\hline & Non-Relative & $381(65.1 \%)$ \\
\hline \multirow[t]{2}{*}{ Number of household Members } & $\leq 4$ & $560(95.7 \%)$ \\
\hline & $\geq 5$ & $25(4.3 \%)$ \\
\hline \multirow[t]{2}{*}{ Household Income/month (Dollar) } & Less 300 Dollar & $295(50.4 \%)$ \\
\hline & $>=300$ Dollar & $290(49.6 \%)$ \\
\hline
\end{tabular}

Table2- Maternal and neonatal anthropometric indices in the studied mothers and infants

\begin{tabular}{|ll|}
\hline Variable (unit) & mean \pm SD \\
\hline Prenatal weight $(\mathrm{kg})$ & $63.40 \pm 11.07$ \\
\hline Mother height $(\mathrm{cm})$ & $160.80 \pm 5.95$ \\
\hline Pre-pregnancy BMI (kg / m 2) & $24.52 \pm 4.12$ \\
\hline Maternal weight gain during pregnancy $(\mathrm{kg})$ & $12.16 \pm 6.85$ \\
\hline Pre-pregnancy energy intake ( Kcal/day) & $2781.30 \pm 1054.37$ \\
\hline Pregnancy(third trimester) energy intake (Kcal/day) & $2755.97 \pm 989.46$ \\
\hline Pre-pregnancy Diversity Score & $5.31 \pm 1.11$ \\
\hline Third trimester of pregnancy DDS & $5.23 \pm 1.42$ \\
\hline Pre-pregnancy MAR & $289.85 \pm 113.12$ \\
\hline Pregnancy MAR pregnant & $371.07 \pm 197.28$ \\
\hline WAZ & $-0.32 \pm 1.08$ \\
\hline HAZ & $0.13 \pm 1.92$ \\
\hline BAZ & $-0.61 \pm 1.48$ \\
\hline HCZ & $-0.11 \pm 1.56$ \\
\hline
\end{tabular}

DDS: Dietary Diversity Scores, MAR: Mean Adequacy Ratio, WAZ: Weight for Age Z-score, HAZ: Height for Age Z-score, BAZ: BMI for Age Z-score, HCZ: Head Circumference for age Z-score

Table3-Mean (standard deviation) of food groups scores according to the Total DDS tertiles of pre-pregnancy and third trimester of gestation of studied mothers 


\begin{tabular}{|c|c|c|c|c|c|c|c|c|}
\hline \multicolumn{5}{|l|}{ Pre-pregnancy } & \multicolumn{4}{|c|}{ Third trimester of pregnancy } \\
\hline & First Tertile & Second Tertile & Third Tertile & $\mathrm{P}$ value $^{1}$ & First Tertile & Second Tertile & Third Tertile & P value ${ }^{1}$ \\
\hline grain score ${ }^{a, b, c}$ & $0.92(0.25)$ & $1.07(0.24)$ & $1.15(0.23)$ & $<0.001$ & $0.66(0.23)$ & $0.79(0.22)$ & $0.98(0.26)$ & $<0 / 001$ \\
\hline vegetable score ${ }^{a, b, c}$ & $0.65(0.30)$ & $0.91(0.29)$ & $1.12(0.29)$ & $<0.001$ & $0.55(0.38)$ & $0.78(0.33)$ & $1.04(0.32)$ & $<0 / 001$ \\
\hline meet score ${ }^{a, b, c}$ & $0.21(0.28)$ & $0.45(0.33)$ & $0.76(0.38)$ & $<0 / 001$ & $0.51(0.38)$ & $0.88(0.39)$ & $1.11(0.40)$ & $<0 / 001$ \\
\hline dairy score ${ }^{a, b, c}$ & $0.71(0.53)$ & $1.01(0.48)$ & $1.40(0.43)$ & $<0 / 001$ & $0.72(0.51)$ & $1.18(0.42)$ & $1.64(0.39)$ & $<0 / 001$ \\
\hline fruit score ${ }^{a, c}$ & $1.76(0.52)$ & $1.98(0.13)$ & $2.00(0.00)$ & $<0 / 001$ & $1.13(0.68)$ & $1.84(0.35)$ & $1.96(0.17)$ & $<0 / 001$ \\
\hline
\end{tabular}

${ }^{1}$ P-value was obtained by one-way ANOVA. The significance between tertiles was obtained by Post-hoc analysis (Tukey, LSD):

a: Significant difference between first and third tertile

b: Significant difference between second and third tertile

c: Significant difference between first and second tertile

Table 4- compared to an average intake of energy, micronutrients and macronutrients for pre-pregnancy and third trimester of pregnancy in mothers in the study * 


\begin{tabular}{|c|c|c|c|c|c|}
\hline \multirow[b]{2}{*}{ P-value* } & \multicolumn{2}{|c|}{ During pregnancy } & \multicolumn{2}{|c|}{ Pre-pregnancy } & \\
\hline & RDA & mean $\pm S D$ & RDA & mean $\pm S D$ & \\
\hline 0.82 & 2855 & $2744.71 \pm 935.46$ & & $2729.6 \pm 895.11$ & Energy(Kcal/d) \\
\hline 0.03 & 175 & $382.08 \pm 140.49$ & & $405.09 \pm 143.12$ & Carbohydrate (g/d) \\
\hline$<0.001$ & 71 & $131.85 \pm 84.47$ & & 99.0336 .11 & Protein (g/d) \\
\hline 0.21 & - & $99.36 \pm 43.88$ & - & $95.41 \pm 39.70$ & Fat $(g / d)$ \\
\hline$<0.001$ & - & $345.85 \pm 226.25$ & - & $25 / 226 \pm 85 / 345$ & Cholesterol(mg/d) \\
\hline 0.85 & - & $29.48 \pm 13.55$ & - & $55 / 13 \pm 48 / 29$ & SFA $(g / d)$ \\
\hline$<0.001$ & - & $20.01 \pm 13.08$ & - & $08 / / 13 \pm 01 / 20$ & PUFA (g/d) \\
\hline$<0.001$ & - & $54.13 \pm 26.80$ & - & $80 / 26 \pm 13 / 54$ & MUFA (g/d) \\
\hline$<0.001$ & 27 & $22.32 \pm 15.64$ & 18 & $64 / 15 \pm 32 / 22$ & Iron (mg/d) \\
\hline$<0.001$ & 1000 & $1407.62 \pm 591.52$ & 1000 & $59 / 591 \pm 62 / 1407$ & Calcium (mg/d) \\
\hline$<0.001$ & 1500 & $4238.09 \pm 1667.52$ & 1500 & $52 / 1667 \pm 09 / 4238$ & Sodium (mg/d) \\
\hline$<0.001$ & 11 & $17.94 \pm 10.62$ & 8 & $62 / 10 \pm 94 / 17$ & Zinc (mg/d) \\
\hline$<0.001$ & 350 & $382.36 \pm 195.85$ & 320 & $85 / 195 \pm 36 / 382$ & Magnesium (mg/d) \\
\hline 0.06 & 700 & $1383.88 \pm 66.49$ & 700 & $49 / 667 \pm 88 / 1383$ & Phosphorus (mg/d) \\
\hline 0.001 & 2900 & $3559.80 \pm 1546.39$ & 2600 & $32 / 1546 \pm 80 / 3559$ & Potassium (mg/d) \\
\hline$<0.001$ & 220 & $0.28 \pm 4.41$ & 150 & $41 / 4 \pm 28 / 0$ & lodine $(\mu \mathrm{g} / \mathrm{d})$ \\
\hline 0.92 & 770 & $1119.09 \pm 860.38$ & 700 & $38 / 860 \pm 09 / 1119$ & Vitamin A ( $\mu \mathrm{g} / \mathrm{d})$ \\
\hline 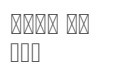 & 1.4 & $2.51 \pm 1.62$ & 1.1 & $62 / 1 \pm 51 / 2$ & Thiamine (mg/d) \\
\hline 0.04 & 1.4 & $25.28 \pm 35.22$ & 1.1 & $22 / 35 \pm 28 / 25$ & Riboflavin (mg/d) \\
\hline$<0.001$ & 1.9 & $1.69 \pm 5.49$ & 1.3 & $4 / 5 \pm 1 / 1$ & Vitamin B6 (mg/d) \\
\hline 0.37 & 600 & $613.15 \pm 247.66$ & 400 & $66 / 247 \pm 15 / 613$ & Folate $(\mu \mathrm{g} / \mathrm{d})$ \\
\hline 0.01 & 2.6 & $8.57 \pm 2.67$ & 2.4 & $6 / 28 \pm 2 / 8$ & Vitamin B12 $(\mu \mathrm{g} / \mathrm{d})$ \\
\hline 0.001 & 85 & $162.43 \pm 122.04$ & 75 & $04 / 122 \pm 43 / 162$ & Vitamin C (mg/d) \\
\hline 0.82 & 15 & $9.90 \pm 8.55$ & 15 & $55 / 21 \pm 90 / 9$ & Vitamin D $(\mu \mathrm{g} / \mathrm{d})$ \\
\hline 0.34 & 15 & $7.99 \pm 7.38$ & 15 & $38 / 7 \pm 99 / 7$ & Vitamin E (mg/d) \\
\hline
\end{tabular}

- Comparison of average intake of energy, micronutrients and macronutrients before pregnancy and the third trimester of pregnancy

RDA: Recommended Dietary Allowance, SFA: Saturated Fatty Acids, PUFA: Polyunsaturated Fatty Acids, MUFA: Monounsaturated Fatty Acids.

Table 5 - Relationship of maternal dietary diversity score and nutritional adequacy before and during pregnancy with anthropometric Measurement of newborn at birth* 


\begin{tabular}{|c|c|c|c|c|c|c|c|c|c|}
\hline & & \multicolumn{4}{|l|}{ DDS } & \multicolumn{4}{|l|}{ MAR } \\
\hline & & \multicolumn{2}{|l|}{ Pre-Pregnancy } & \multicolumn{2}{|c|}{ Third Trimester of Pregnancy } & \multicolumn{2}{|l|}{ Pre-Pregnancy } & \multicolumn{2}{|c|}{ Third Trimester of Pregnancy } \\
\hline & & $\mathrm{B}(\mathrm{Cl})$ & P-value & $\mathrm{B}(\mathrm{Cl})$ & $\begin{array}{l}\mathrm{P}- \\
\text { value }\end{array}$ & $\mathrm{B}(\mathrm{Cl})$ & $\begin{array}{l}\mathrm{P} \text { - } \\
\text { value }\end{array}$ & $\mathrm{B}(\mathrm{Cl})$ & $\begin{array}{l}\mathrm{P}- \\
\text { valu }\end{array}$ \\
\hline \multirow[t]{2}{*}{ Weight } & $\begin{array}{l}\text { Crude } \\
\text { model }^{1}\end{array}$ & 18.16(-22.32_58.64) & 0.37 & $-14.36\left(-51.48 \_22.76\right)$ & 0.44 & -29.34(-83.73_25.04) & 0.29 & $-53.5\left(-116.2 \_9.12\right)$ & 0.09 \\
\hline & $\begin{array}{l}\text { Adjusted } \\
\text { Model }^{2}\end{array}$ & $-2.65\left(-74.00 \_68.70\right)$ & 0.94 & -76.93(-142.18_11.68) & 0.02 & $-0.54\left(-1.09 \_0.004\right)$ & 0.05 & $-98.51(-181.94-15.08)$ & 0.02 \\
\hline \multirow[t]{2}{*}{ Height } & $\begin{array}{l}\text { Crude } \\
\text { model }^{1}\end{array}$ & $0.03\left(-0.15 \_0.21\right)$ & 0.73 & 0.008(-0.18_0.16) & 0.92 & $-0.17\left(-0.41 \_0.07\right)$ & 0.17 & $-0.22\left(-0.51 \_0.07\right)$ & 0.14 \\
\hline & $\begin{array}{l}\text { Adjusted } \\
\text { Model }^{2}\end{array}$ & 0.02(-0.26_0.31) & 0.86 & $-0.009\left(-0.3 \_0.28\right)$ & 0.95 & $-0.001\left(-0.004 \_0.001\right)$ & 0.27 & $-0.38\left(-0.76 \_0.003\right)$ & 0.05 \\
\hline \multirow[t]{2}{*}{$\mathrm{CH}$} & $\begin{array}{l}\text { Crude } \\
\text { model }^{1}\end{array}$ & $0.06\left(-0.09 \_0.23\right)$ & 0.40 & $0.04\left(-0.11 \_0.2\right)$ & 0.57 & $-0.28(-0.5-0.07)$ & 0.009 & $-0.17\left(0.44 \_0.09\right)$ & 0.21 \\
\hline & $\begin{array}{l}\text { Adjusted } \\
\text { Model }^{2}\end{array}$ & 0.13(-0.02_0.29) & 0.25 & $0.06\left(-0.24 \_0.36\right)$ & 0.67 & $-0.25\left(-0.59 \_0.07\right)$ & 0.12 & $-0.36\left(0-.8 \_0.07\right)$ & 0.10 \\
\hline \multirow[t]{2}{*}{ WAZ } & $\begin{array}{l}\text { Crude } \\
\text { model }^{1}\end{array}$ & $0.04\left(-0.04 \_0.13\right)$ & 0.28 & $-0.01\left(-0.09 \_0.06\right)$ & 0.71 & $-0.08\left(-0.2 \_0.04\right)$ & 0.19 & $-0.09\left(-0.23 \_0.04\right)$ & 0.17 \\
\hline & $\begin{array}{l}\text { Adjusted } \\
\text { Model }^{2}\end{array}$ & $0.002\left(0.15 \_0.15\right)$ & 0.98 & $-0.16\left(-0.23 \_0.3\right)$ & 0.02 & $-0.001\left(-0.002 \_0.00\right)$ & 0.03 & $-0.18(-0.35-0.004)$ & 0.04 \\
\hline \multirow[t]{2}{*}{ HAZ } & $\begin{array}{l}\text { Crude } \\
\text { model }^{1}\end{array}$ & $0.009\left(-0.08 \_0.1\right)$ & 0.85 & $-0.01\left(-0.11 \_0.08\right)$ & 0.76 & $-0.08\left(-0.21 \_0.04\right)$ & 0.21 & $-0.04\left(-0.2_{-}-.12\right)$ & 0.76 \\
\hline & $\begin{array}{l}\text { Adjusted } \\
\text { Model }^{2}\end{array}$ & $0.01\left(0.14 \_0.16\right)$ & 0.86 & $-0.003\left(-0.16 \_0.15\right)$ & 0.96 & $0.71\left(-0.002 \_0.00\right)$ & 0.2 & $-0.14\left(-0.34 \_0.06\right)$ & 0.86 \\
\hline \multirow[t]{2}{*}{ BAZ } & $\begin{array}{l}\text { Crude } \\
\text { model }^{1}\end{array}$ & $0.07\left(-0.04 \_0.2\right)$ & 0.21 & $-0.01\left(-0.12 \_0.1\right)$ & 0.85 & $-0.05\left(-0.22 \_0.1\right)$ & 0.49 & $-0.11\left(-0.3 \_0.07\right)$ & 0.85 \\
\hline & $\begin{array}{l}\text { Adjusted } \\
\text { Model }^{2}\end{array}$ & $0.008\left(-0.2 \_0.21\right)$ & 0.93 & $-0.24\left(0.41_{-}-0.02\right)$ & 0.007 & $-0.001\left(-0.003 \_0.00\right)$ & 0.12 & $-0.16\left(-0.4 \_0.07\right)$ & 0.08 \\
\hline \multirow[t]{2}{*}{$\mathrm{HCZ}$} & $\begin{array}{l}\text { Crude } \\
\text { model }^{1}\end{array}$ & $0.1\left(-0.01 \_0.05\right)$ & 0.09 & $0.04\left(-0.08 \_0.17\right)$ & 0.47 & $-0.001\left(-0.002 \_0.001\right)$ & 0.24 & $-0.09\left(-0.06 \_0.07\right)$ & 0.22 \\
\hline & $\begin{array}{l}\text { Adjusted } \\
\text { Model }^{2}\end{array}$ & 0.03(0.19_0.25) & 0.78 & $-0.04(-0.06-0.42)$ & 0.07 & $-0.01\left(-0.002 \_0.01\right)$ & 0.1 & $-0.001\left(-0.44 \_0.11\right)$ & 0.19 \\
\hline
\end{tabular}

P-value was obtained using linear regression analysis and $\mathrm{P}<0.05$ was considered as significant

B: Regression coefficient, Cl: Confidence Intervals

HC: Head Circumference WAZ: Weight for Age Z-score, HAZ: Height for Age Z-score, BAZ: BMI for Age Z-score, HCZ: Head Circumference for age Z-score

${ }^{1}$ Row Model: without adjustment of confounding factors

${ }^{2}$ Adjusted model: Adjusted for maternal confounding variables (age, education, occupation, iron and folic acid supplementation status, smoking, maternal illness, pregnancy complications, number of births, history of miscarriage and stillbirth) and infant-related confounding variables (gender, polygamy, delivery time).

\section{Figures}




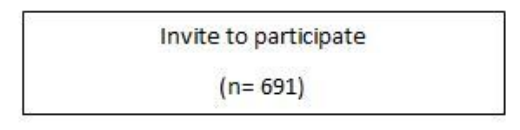

Mothers with available FFQ data:

$(n=585)$

Mothers with available $24-\mathrm{h}$ recall data:

$(n=345)$

Infants Information at birth:

$(n=337)$
Not included due to: not willing to

cooperate

( $n=106)$

Excluded due to:

Loss of pregnancy or miscarriage $(n=21)$

No access to maternal, Migration $(n=219)$

Excluded due to:

Infant death $(n=3)$

No access to electronic health record

$(n=2)$, migration $(n=3)$

\section{Figure 1}

Flow chart of the recruitment process 